\title{
New Versions of the Hermite Bieler Theorem in Stability Contexts
}

\author{
Ziad Zahreddine \\ Mathematics Division, College of Science \& Information Systems, Rafik Hariri University, Mechref, Damour, Lebanon \\ Email address: \\ zahreddinezn@rhu.edu.lb, ziadzahr@hotmail.com

\section{To cite this article:} \\ Ziad Zahreddine. New Versions of the Hermite Bieler Theorem in Stability Contexts. American Journal of Applied Mathematics. \\ Vol. 7, No. 1, 2019, pp. 1-4. doi: 10.11648/j.ajam.20190701.11
}

Received: January 3, 2019; Accepted: January 24, 2019; Published: February 25, 2019

\begin{abstract}
The Hermite-Bieler theorem played key roles in several control theory problems including the proof of Kharitonov's theorem and derivations of elementary proofs of the Routh's algorithm for determining the Hurwitz stability of a real polynomial. In the present work, we explore the stability of complex continuous-time systems of differential equations. Using the theory of positive paraodd functions, we obtain Hermite-Bieler like conditions for the Routh-Hurwitz stability of such systems. We also look at the problem of stability of discrete-time systems of difference equations. By using suitable conformal mappings, we also establish Hermite-Bieler like conditions for the Schur-Cohn stability of these systems. In both cases, the conditions are necessary as well as sufficient.
\end{abstract}

Keywords: Hermite-Bieler Theorem, Routh-Hurwitz Criterion, Schur-Cohn Criterion, Stability Analysis, Positive Para-Odd Functions, Conformal Mappings

\section{Introduction}

The Routh-Hurwitz criterion for the stability of continuous-time systems of differential equations, and the Schur-Cohn criterion for the stability of discrete-time systems of difference equations have long been treated. The Routh-Hurwitz criterion settles the stability of continuoustime systems with real coefficients. The Extended Routh Array (ERA) which proved to be a natural extension of the Routh Array was introduced [13]. The ERA settles the asymptotic stability of systems with complex coefficients which arise in modern communication, information theory, signal analysis and detection. The main argument in the construction of the ERA dwells on the use of positive paraodd functions which continue to play a highly effective role in the stability of complex continuous-time systems. The Schur-Cohn criterion settles the stability of discrete-time systems, see for example [2]. Using conformal mappings, explicit relationships between the Routh-Hurwitz and the Schur-Cohn criteria were established, [14]. Recently, further connections between the two stability types were explored, [10]. Postnikov gave rich contributions in these directions, including Cauchy indices, Sturm chains, amplitude-phase interpretation of stability, principle of the argument, continued fractions, and the Hermite-Biehler theorem [6].

Kharitonov's famous theorem relied heavily on the Hermite-Bieler theorem [4]. That led to tremendous interest in the latter which also provided elementary derivations of Routh's algorithm for determining the Hurwitz stability of a real polynomial. A version of the Hermite-Bieler theorem was used to determine the set of all stabilizing proportionalintegral PI controllers [1]. Using similar approaches, results on the stability of fractional order polynomials are obtained [7]. By means of the square transform, a class of symmetric indefinite Hermite-Bieler functions was obtained from positive definite ones [5]. The Hermite-Bieler theorem was also used to study the real-rootedness of Eulerian polynomials of different types [8]. Some of the author's contributions were done in the spirit of Kharitonov's theorem $[11,12]$.

In this paper, we provide further characterizations of the Routh-Hurwitz and the Schur-Cohn stability criteria in terms of the Hermite-Bieler theorem.

\section{Routh-Hurwitz Case}

We recall the definitions of [9].

Definition 2.1 
A non-constant polynomial is a Hurwitz polynomial if all its roots have negative real parts.

Definition 2.2

The paraconjugate of a rational function $f(z)$ is defined by $f^{*}(z)=\overline{f(-\bar{z})}$, where $\bar{z}$ denotes the complex conjugate of $z$.

For example, if

$$
f(z)=z^{n}+a_{1} z^{n-1}+\cdots+a_{n-2} z^{2}+a_{n-1} z+a_{n}
$$

Then

$$
f^{*}(z)=(-1)^{n} z^{n}+(-1)^{n-1} \bar{a}_{1} z^{n-1}+\cdots+\bar{a}_{n-2} z^{2}-\bar{a}_{n-1} z+\bar{a}_{n}
$$

\section{Definition 2.3}

A rational function $\mathrm{g}$ is said to be positive if $\operatorname{Re} g(z)>0$ where $\operatorname{Re} z>0$.

Definition 2.4

A rational function $h$ is called para-odd if it satisfies $h^{*}=-h$, where ${ }^{*}$ denotes paraconjugation.

Therefore, if $\mathrm{h}$ is positive and satisfies $h^{*}=-h$, we say that $\mathrm{h}$ is positive para-odd.

Let $f(z)$ be as defined in (1) and let it represent the characteristic polynomial of a continuous-time system of differential equations.

Define the function

$$
h(z)= \begin{cases}\frac{f-f^{*}}{f+f^{*}} & \text { if } n \text { odd } \\ \frac{f+f^{*}}{f-f^{*}} & \text { if } n \text { even }\end{cases}
$$

Obviously, $h$ can be written as

$$
h(z)=\frac{z^{n}+i \operatorname{Im} a_{1} z^{n-1}+\operatorname{Re} a_{2} z^{n-2}+i \operatorname{Im} a_{3} z^{n-3}+\operatorname{Re} a_{4} z^{n-4}+\cdots}{\operatorname{Re} a_{1} z^{n-1}+i \operatorname{Im} a_{2} z^{n-2}+\operatorname{Re} a_{3} z^{n-3}+i \operatorname{Im} a_{4} z^{n-4} \cdots}
$$
[3].

This function is sometimes referred to as the test fraction, The following theorem was established in [13].

Theorem 2.1 ([13, Theorem 3.1])

The polynomial $f(z)=z^{n}+a_{1} z^{n-1}+\cdots+a_{n-2} z^{2}+a_{n-1} z+a_{n}$ is Hurwitz if and only if the test fraction $h$ can be written in the partial fraction expansion form

$$
h(z)=a_{0}+d_{0} z+\frac{d_{1}}{z-i w_{1}}+\frac{d_{2}}{z-i w_{2}}+\cdots+\frac{d_{n-1}}{z-i w_{n-1}}
$$

Where $\operatorname{Re} a_{0}=0$, and $d_{j}>0$ for $0 \leq j \leq n-1$.

Let $N(z)$ and $D(z)$ denote respectively the numerator and denominator of $h(z)$ as defined in (3).

Lemma 2.1

The function $1 / g$ is positive if and only if $g$ is positive.

Proof

For any nonzero complex number $z$, it is clear that

$$
\frac{1}{z}=\frac{\bar{z}}{|z|^{2}}
$$

Therefore, for such $z, \operatorname{Re}(z)$ and $\operatorname{Re}(1 / z)$ have the same sign, from which it follows that $1 / g$ is positive if and only if $g$ is positive.

The following is a new version of the Hermite-Bieler theorem in the Routh-Hurwitz stability context.

Theorem 2.2

The polynomial $f(z)=z^{n}+a_{1} z^{n-1}+\cdots+a_{n-2} z^{2}+a_{n-1} z+a_{n}$ is Hurwitz if and only if $\operatorname{Re} a_{1}>0$, and the zeros of $N$ and $D$ are simple and lie on the imaginary axis. Furthermore, the zeros of $N$ interlock with those of $D$.

Proof

Assume that $f$ is Hurwitz, then by Theorem 2.1 above, $h(z)$ can be written as

$$
h(z)=a_{0}+d_{0} z+\frac{d_{1}}{z-i w_{1}}+\frac{d_{2}}{z-i w_{2}}+\cdots+\frac{d_{n-1}}{z-i w_{n-1}}
$$

Where $\operatorname{Re} a_{0}=0$, and $d_{j}>0$ for $0 \leq j \leq n-1$.

Obviously, $a_{0}+d_{0} z$ is the quotient obtained upon division of $N$ by $D$. It follows that $d_{0}=1 / \operatorname{Re} a_{1}$ which in turn implies that $\operatorname{Re} a_{1}>0$.

Also the partial fraction expression of $h(z)$ shows that the zeros of $D$ (denominator of $h$ ) are simple and imaginary.

By Lemma 2.1, $h$ is positive if and only if $1 / h$ is positive. Therefore, and for a similar reason, the zeros of the denominator of $1 / h$, i.e. the zeros of $N$ are also simple and imaginary.

We may assume that $a_{0}=0$. Otherwise, we define the function $k=h-a_{0}$, and we continue with $k$.

Let $r(t)=-i h(i t)$, then $r(t)$ can be written as

$$
r(t)=d_{0} t-\frac{d_{1}}{t-w_{1}}-\frac{d_{2}}{t-w_{2}}-\cdots-\frac{d_{n-1}}{t-w_{n-1}}
$$

It follows that

$$
r^{\prime}(t)=d_{0}+\frac{d_{1}}{\left(t-w_{1}\right)^{2}}+\frac{d_{2}}{\left(t-w_{2}\right)^{2}}+\cdots+\frac{d_{n-1}}{\left(t-w_{n-1}\right)^{2}}
$$

leading to $r^{\prime}(t)>0$ for $t \neq w_{k}$.

Furthermore, on each interval $\left(w_{k}, w_{k+1}\right)$ the function $r(t)$ increases from $-\infty$ to $\infty$.

It follows that the equation $r(t)=0$ has only one root on each such interval and that completes the first part of the proof.

Conversely, assume $i t_{1}, i t_{2}, \ldots, i t_{n}$ are the roots of $N(z)$, and $i w_{1}, i w_{2}, \ldots, i w_{n}$ the roots of $D(z)$ such that.

$$
t_{1}<w_{1}<t_{2}<w_{2}<\cdots<t_{n-1}<w_{n-1}<t_{n}
$$

Consider the partial fraction expansion of $h$ 


$$
h(z)=a_{0}+d_{0} z+\frac{d_{1}}{z-i w_{1}}+\frac{d_{2}}{z-i w_{2}}+\cdots+\frac{d_{n-1}}{z-i w_{n-1}}
$$

Obviously $d_{0}=1 / \operatorname{Re} a_{1}$, and since $\operatorname{Re} a_{1}$ is now assumed to be positive, it follows that $d_{0}>0$.

Again, we may assume that $a_{0}=0$.

Reconsider the function

$$
r(t)=-i h(i t)=d_{0} t-\frac{d_{1}}{t-w_{1}}-\frac{d_{2}}{t-w_{2}}-\cdots-\frac{d_{n-1}}{t-w_{n-1}}
$$

From the set of inequalities $t_{1}<w_{1}<t_{2}<w_{2}<\cdots<t_{n-1}<w_{n-1}<t_{n}$ it follows that $d_{k}>0$ for $k=1, \ldots, n$.

Therefore, the polynomial

$f(z)=z^{n}+a_{1} z^{n-1}+\cdots+a_{n-2} z^{2}+a_{n-1} z+a_{n}$ is Hurwitz by

Theorem 2.1 above.

\section{Schur-Cohn Case}

We make the following definitions

\section{Definition 3.1}

A non-constant polynomial is a Schur polynomial if all its roots lie inside the unit disc.

Definition 3.2

Consider the polynomial

$$
g(s)=s^{n}+a_{1} s^{n-1}+\cdots+a_{n-1} s+a_{n}
$$

And let it represent the characteristic polynomial of a discrete-time system of difference equations.

The reciprocal of $g$ is defined by

$$
g^{\tau}(s)=s^{n} \overline{g(1 / \bar{s})}=\bar{a}_{n} s^{n}+\bar{a}_{n-1} s^{n-1}+\cdots+\bar{a}_{1} s+\bar{a}_{0}
$$

The Schur-Cohn counterpart to Theorem 2.2 is now given. A part of the proof is based on the techniques adopted in proving Theorem 3 of [9].

Theorem 3.1

The polynomial $g(s)=s^{n}+a_{1} s^{n-1}+\cdots+a_{n-1} s+a_{n} \quad$ is Schur-Cohn stable if and only if $\left|a_{n}\right|<1$ and the zeros of $g-g^{\tau}$ and $g+g^{\tau}$ are simple and lie on the unit circle. Furthermore, the zeros of $g-g^{\tau}$ interlock with those of $g+g^{\tau}$.

Proof

The conformal mapping $s=\frac{1+z}{1-z}$ maps the imaginary $z$ axis onto the $s$-unit circle.

In other words: $\operatorname{Re} z<0 \Leftrightarrow|s|<1$

Introduce the function $f(z)=(1-z)^{n} g\left(\frac{1+z}{1-z}\right)$
It follows that $f(z)=\sum_{k=0}^{n} a_{k}(1-z)^{k}(1+z)^{n-k}$

Since $s=\frac{1+z}{1-z}$, the polynomial $f(z)$ is Routh-Hurwitz if and only if $g(s)$ is Schur-Cohn.

The paraconjugate of $f$ is given by

$$
f^{*}(z)=\sum_{k=0}^{n} \bar{a}_{k}(1+z)^{k}(1-z)^{n-k}
$$

Therefore,

$$
(1-z)^{n} g^{\tau}\left(\frac{1+z}{1-z}\right)=\sum_{k=0}^{n} \bar{a}_{k}(1+z)^{k}(1-z)^{n-k}
$$

Leading to

$$
f^{*}(z)=(1-z)^{n} g^{\tau}\left(\frac{1+z}{1-z}\right)
$$

From the above expressions of $f$ and $f^{*}$, we get

$$
f(z)+f^{*}(z)=(1-z)^{n}\left[g\left(\frac{1+z}{1-z}\right)+g^{\tau}\left(\frac{1+z}{1-z}\right)\right]
$$

And

$$
f(z)-f^{*}(z)=(1-z)^{n}\left[g\left(\frac{1+z}{1-z}\right)-g^{\tau}\left(\frac{1+z}{1-z}\right)\right]
$$

The fact that $\operatorname{Re} z<0 \Leftrightarrow|s|<1$ implies that the following two propositions are equivalent:

$\mathrm{P}_{1}$ : The zeros of $f+f^{*}$ and $f-f^{*}$ are simple, lie on the imaginary axis and interlock.

$\mathrm{P}_{2}$ : The zeros of $g+g^{\tau}$ and $g-g^{\tau}$ are simple, lie on the unit circle and interlock.

It should be noted that $f+f^{*}$ and $f-f^{*}$ are respectively the numerator $N$ and denominator $D$ of $h$ as defined in (2) in case $n$ is even, and the other way round if $n$ is odd.

To complete the proof, we need to write the function $f$ in the following form:

$$
f(z)=\sum_{k=0}^{n} b_{k} z^{n-k}
$$

Then by applying Theorem 3.1 with $p=1$, or Theorem 3.2 with $p=n$ [14], we get

$\operatorname{Re} b_{1}<0 \Leftrightarrow\left|a_{n}\right|<1$,

And that completes the proof.

\section{Conclusion}

Two important problems in stability theory were 
considered, the Routh-Hurwitz stability problem for continuous-time systems of differential equations and the Schur-Cohn stability problem for discrete-time systems of difference equations. In both cases, we establish HermiteBieler like conditions which guarantee the stability of the system. The conditions are necessary as well as sufficient. In the continuous-time case, we invoked the theory of positive para-odd functions which proved highly effective in stability analysis. In the discrete-time case, the use of suitable conformal mappings permits a smooth transfer of results from the continuous to the discrete case.

\section{References}

[1] S. Elmadssia, K. Saadaoui, M. Benrejeb, PI controller design for time delay systems using an extension of the HermiteBiehler theorem, Journal of Industrial Engineering Volume 2013, Article ID 813037, 6 pages.

[2] G. Heinig, U. Jungnickel, On the Routh-Hurwitz and SchurCohn Problems for Matrix Polynomials and Generalized Bezoutians, Math. Nachr.11G (1984), 185-196.

[3] R. Hovstad, A short proof of continued fraction test for the stability of polynomials, Pro. Amer. Math. Soc., 105, No. 1 (1989), 76-79.

[4] V. L. Kharitonov, The asymptotic stability of the equilibrium state of a family of systems of linear differential equations, Differentsial'nye Uravneniya 14 (1978), no. 11, 2086-2088.

[5] V. Pivovarchik, H. Woracek, The square-transform of hermitebiehler functions. a geometric approach, Methods of Functional Analysis and Topology, Vol. 13 (2007), no. 2, pp. 187-200.
[6] M. M. Postnikov, Stable polynomials, Moscow, Nauka, 1981 (in Russian).

[7] B. Senol, C. Yeroglu, N. Tan, Analysis of fractional order polynomials using Hermite-Biehler theorem, International Conference on Fractional Differentiation and Its Applications 2014, 1-5, 2014.

[8] L. B. Yang, P. B. Zhang, The real-rootedness of Eulerian polynomials via the Hermite-Biehler Theorem, Discrete Mathematics and Theoretical Computer Science proc., FPSAC'15, 2015, 465-474 Nancy, France.

[9] Z. Zahreddine, On Positive Para-odd and Complex Discrete Reactance Functions, Journal of Interdisciplinary Mathematics, Taylor \& Francis, Vol. 21, 2018 - Issue 1, 243251.

[10] Z. Zahreddine, Parallel Properties of Poles of Positive Functions and those of Discrete Reactance Functions, International Journal of Mathematical Analysis. Vol 11, 2017, no. $24,1141-1150$.

[11] Z. Zahreddine, Alternative Approaches to the General Stability Problem, Conference in Mathematical Analysis \& Applications, American University of Sharjah, 2003.

[12] Z. Zahreddine, On the $\Gamma$-stability of systems of differential equations in the Routh-Hurwitz and Schur-Cohn cases, Bulletin of the Belgian Mathematical Society, 3 (1996), 363368 .

[13] Z. Zahreddine, An extension of the Routh array for the asymptotic stability of a system of differential equations with complex coefficients, Applicable Analysis, 49 (1993), 61-72.

[14] Z. Zahreddine, Explicit relationships between Routh-Hurwitz and Schur-Cohn types of stability, Bulletin of the Irish Mathematical Society, 29 (1992), 49-54. 